\title{
GEOMETRIA ACTIVA Y GEOMETRIA DE LAS TRANSFORMACIONES
}

\author{
Carlos E. Vasco U. \\ Profesor \\ Departamento de Matemáticas y Estadística \\ Universidad Nacional de Colombia
}

\section{Introducción}

Me propongo en este artículo sólo dos modestos objetivos: contrastar la geometría activa que se propone en el nuevo currículo de matemáticas del Ministerio de Educación Nacional con la geometría del llamado "Programa de Erlangen" de Félix Klein, y con la llamada "Geometría de las Transformaciones".

Tengo en primer lugar que justificar estos dos tipos de contrastación. Supongo que se conoce, así sea de paso, el enfoque de los programas de matemáticas de la renovación curricular, que están publicados desde 1984 por el Ministerio de Educación, [8], y que han salido impresos también al comienzo de los nuevos programas de $6^{\circ}, 7^{\circ}$ y $8^{\circ}$ grado, [9], [10], [11], y supongo que en particular se conoce el enfoque de la geometría como exploración activa del espacio y de los modos de representación del mismo en la imaginación y en el plano del dibujo.

Al hablar de "geometría activa" en los nuevos programas del Ministerio, he comprobado que pueden surgir, y de hecho surgen, los dos tipos de equívocos que me propongo despejar. El primer equivoco surge de la confusión de la geometría activa con el enfoque del "Programa de Erlangen", como si se estuviera proponiendo dejar de lado la métrica del espacio y empezar con la topología como geometría de las deformaciones continuas y sus invariantes, pasar luego a la geometría proyectiva, a la afín y sólo al final a la euclidiana. El segundo equívoco surge de la confusión de la geometría activa con el enfoque de la llamada "Geometría de las Transformaciones", propuesta por la Matemática Moderna, y en particular por Marshall Stone al final de la década de los cincuenta y comienzos de la de los sesenta; esta geometría empezaba con el estudio de las reflexiones sobre una recta dada en el plano, a partir de las cuales se definían las rotaciones y las translaciones, para continuar luego con el estudio de todo el grupo de isometrías del plano euclidiano.

Desafortunadamente estas confusiones han servido para que algunas personas duden de la conveniencia de adoptar los nuevos programas de matemáticas, y para que otras lleguen a atacarlos abiertamente como demasiado formales, o hasta a calificarlos de anticuados en más de 20 años por no haberse aceptado en esos programas el fracaso de la Matemática Moderna.

Lo que sucede es que cada uno de nosotros, y en particular el lector, tiene todo el derecho de proyectar en la expresión geometría activa" lo que esta expresión le despierte espontáneamente en su cerebro, y eso es inevitable.

Si se aceptan los resultados de la psicología genética, la tendencia a la asimilación de lo nuevo a las estructuras antiguas que uno ya tiene, es el comportamiento inicial más característico del conocimiento humano. 
Lo único que pido al lector es que detenga un momento el juicio proyectivo o asimilativo inicial que se haya hecho acerca de lo que es la geometría activa propuesta en los programas de la renovación curricular, y que preste atención a los argumentos que podrían hacerle modificar esa preconcepción, para ver si es conveniente acomodarla a la concepción de geometría activa que propongo, más bien que tratar de asimilar la propuesta de geometría activa a la propia preconcepción.

Para profundizar un poco en el tema, puede leerse el capítulo "Sistemas geométricos" de mi trabajo de ascenso a Profesor Titular de la Universidad Nacional, capítulo que salió publicado en el segundo volumen del libro "Un nuevo enfoque para la didáctica de las matemáticas", [14].

\section{La geometría activa}

La idea de geometría activa que propongo para los nuevos programas de matemáticas vino de mi preocupación por la falta de habilidades de manejo mental y gráfico del espacio por parte de los estudiantes que llegaban a las universidades Javeriana y Nacional en la década de los setenta. A pesar de que los programas del Decreto 080 de 1974 tenían unidades de geometría euclidiana, muchos profesores no enseñaban esas unidades, ya sea por falta de tiempo, ya por el desprecio a la geometría euclidiana que venía del grupo Bourbaki. La arenga de Dieudonné en el coloquio de Royaumont en 1959 había resonado en todo el mundo, y había tenido eco también en nuestra patria: "Muera Euclides!" (ver por ejemplo [6]; en español puede leerse el reciente libro de Angel Ruiz [13]).

Mi inspiración vino de dos fuentes: una en Colombia y otra en los Estados Unidos. Aquí en Colombia encontré que el Profesor Alberto Campos estaba trabajando muy seriamente en resucitar la moribunda geometría, tanto en la carrera de matemáticas de la Universidad Nacional como en la carrera de filosofía. Tuve la fortuna de que por mis aficiones filosóficas, el Profesor Campos me pidiera colaborar en la conformación de los programas de las cuatro asignaturas de Lógica y Matemática que se proponen a los estudiantes de la carrera de filosofía en la Universidad Nacional. El primer curso seguía la historia de las matemáticas griegas desde los presocráticos hasta Euclides, y el segundo tomaba las geometrías desde Euclides hasta Hilbert y Bourbaki, pasando por Kant y las geometrías no euclidianas. Me interesé muchísimo por esos temas, y aprendí mucho más enseñando esos dos cursos siguiendo las notas escritas por el Profesor Campos, [1], [2], y el comentario a los Elementos de Euclides escrito por Heath en la colección Dover [5].

Descubrí allí que la geometría de Euclides tenía un horror a primera vista inexplicable por los movimientos. Todas las relaciones eran estáticas: el paralelismo, la perpendicularidad, la congruencia y la semejanza. Las demostraciones se hacían por prolongaciones y cortes para evitar mover las figuras. La explicación era la corriente platónica, que despreciaba el movimiento como perteneciente al mundo de lo mutable, al mundo de la opinión, y por lo tanto muy alejado del verdadero mundo de la ciencia, el mundo de lo inmutable y lo eterno. Cuando se atrevió Euclides a deslizar un triángulo para hacerlo coincidir con otro con el fin intentar probar los tres casos de congruencia de dos triángulos, las demostraciones fallan catastróficamente. Cualquier intento de repararlas conduce al fracaso, lo cual obligó a Hilbert a agregar una de ellas como un axioma más a la ya extensa lista de los axiomas hilbertianos.

Sin embargo, en la definición de congruencia de una figura con otra, relación llamada allí de "igualdad", si miramos la raíz griega ("epipipto"), Euclides habla claramente de que 
la segunda figura "caiga bien encima" de la primera. Noté también que la extraña definición de recta de Euclides tenía sentido si uno trata de deslizar o girar una recta sobre sí misma: trátese de hacer rodar un alambre recto sobre una superficie plana: si es bien recto, cae bien sobre cualquiera de sus puntos. El misterioso postulado cuarto sobre la igualdad de todos los ángulos rectos parece recobrar el sentido si se va rotando una recta sobre otra hasta que los cuatro ángulos sean iguales de amplios: en ese momento son rectos. No es pues que todos los ángulos rectos sean iguales, lo cual es tautológico, sino que cuando todos los ángulos son iguales, son rectos. Así me fui acercando a la geometría clásica a través de los giros, las traslaciones, los deslizamientos, las reflexiones, las ampliaciones y las reducciones. Esa fue la fuente colombiana de mi afición por la geometría activa.

En los Estados Unidos conocí los estudios que se realizaban en el MIT por parte de Seymour Papert.

La ilusión de que el lenguaje LOGO iba a resolver todos los problemas de la educación matemática era claramente eso: una ilusión. Pero las ideas de Papert sobre la geometría que él llamaba "sintónica con el cuerpo" ("body-syntonic"); la idea de imaginar los movimientos de la tortuga en el plano poniéndose uno mismo a moverse sobre el piso, siguiendo las instrucciones de otro; la idea de aprovechar la experiencia de navegación en yate que tenían los niños ricos de Boston, así no hubiera un solo niño en las escuelas de Bogotá que la tuviera, me inspiraron sobre la necesidad de partir de los movimientos del propio cuerpo y de los objetos y juguetes más sencillos como el verdadero comienzo sintónico de la geometría (ver [12]).

Es conocida la anécdota que cuento con frecuencia sobre las medias vueltas y cuartos de vuelta: un niño de primer grado en clase de educación física en uno de los barrios marginados sabía cuanto era la mitad de un cuarto de vuelta; pude pues ver allí un primer concepto geométrico de ángulo activo, y pude ver los fraccionarios como operadores sintónicos con el cuerpo del niño, dos años antes de que se los enseñaran en clase con el contraproducente método de partir objetos en dos mitades.

Hice algunos talleres de geometría activa a partir de los movimientos del cuerpo. Recuerdo uno de ellos en Pereira. Percibí una gran resistencia inicial, hasta que me atreví a citar al patio principal del colegio a todos los profesores, en el segundo piso los que sólo quisieran mirar, y en el primero a los que quisieran participar. Bailar las rotaciones, los ángulos internos y externos de los triángulos y los cuadriláteros, bailar las traslaciones y los deslizamientos, fueron actividades que rompieron el hielo y empezaron a cambiar las relaciones de los profesores conmigo y con la geometría. Después me contaron que la resistencia inicial se debía a que un profesor de Bogotá había ido el año anterior a darles un curso que también se llamaba "geometría activa", pero que después del discurso inicial en favor de la actividad y en contra de las figuras estáticas en el tablero, todo el resto del curso no había hecho sino pintar más y más figuras estáticas en el tablero.

Noté a partir de esos talleres con maestros, que no sólo los niños tienen dificultades en manejar el movimiento de sus cuerpos e interiorizar las operaciones geométricas en sus cerebros. Hay algunos ejercicios de gimnasia que nos permiten caer en la cuenta de esa falta de geometría activa en nuestros años escolares. Con Celia Castiblanco, Cecilia Casasbuenas y Teresa León hemos diseñado muchas actividades para que los niños que las sigan no tengan esa limitación física y mental de falta de geometría muscular, de geometría de la imaginación y de geometría del dibujo. 
A partir de esas dos inspiraciones, y con la ayuda teórica del marco general de las matemáticas que había propuesto para los nuevos programas del Ministerio, empecé a desarrollar más sistemáticamente la propuesta de la geometría activa como exploración del espacio y de sus modos de representación en la imaginación y en el plano.

El enfoque de los programas me hacía ver claramente la distinción entre las operaciones o transformaciones y las relaciones o correspondencias. Las primeras son más dinámicas y se refieren a la práctica; las segundas son más estáticas y se refieren a la teoría.

Parecía que los sistemas geométricos clásicos tenían sólo relaciones estáticas. Pero las relaciones se establecen por medio de operaciones, y para completar los sistemas geométricos había entonces que reconstruir las operaciones en la geometría euclidiana. Llegué por esa vía a las traslaciones, las rotaciones y las reflexiones, y luego a las ampliaciones y reducciones que llamamos con el alienante y poco atractivo nombre de "homotecias".

Noté también al hacer el análisis de los distintos temas de las matemáticas escolares por medio de la teoría de sistemas, que los sistemas geométricos eran claramente distintos de los métricos, pero no en el sentido de que los geométricos sin métrica euclidiana llevaran a la topología y al "Programa de Erlangen", sino en el sentido más sencillo de que aun la geometría euclidiana podía y debía distinguirse claramente de los sistemas métricos en el sentido de sistemas de medidas con asignaciones numéricas.

Por ejemplo, claramente un segmento se distingue como objeto geométrico de su longitud, que es un concepto métrico, así para distinguir un segmento recto de uno curvo se necesite una métrica subyacente; una región bien delimitada se distingue como objeto geométrico de su área, que es un concepto métrico, así para distinguir una región plana de una curva se necesite una métrica subyacente; un cuerpo sólido se distingue de su volumen, siendo el uno un objeto geométrico y el otro un concepto métrico; un ángulo como bilátero geométrico (ya sea formado por dos segmentos o por dos semirrectas con un vértice común) se distingue claramente de la amplitud angular, concepto métrico que permite determinar al menos cuatro amplitudes orientadas diferentes y dos amplitudes no orientadas diferentes en cada bilátero, excepto en el caso de que los dos segmentos o semirrectas sean opuestos, pues en ese caso las dos amplitudes no orientadas resultan de igual medida angular.

De esas distinciones entre los sistemas geométricos y los sistemas métricos, y dentro de los sistemas geométricos de los elementos, en este caso las figuras, las operaciones o transformaciones, y las relaciones, provino la propuesta de geometría activa como exploración activa del espacio.

\section{El Programa de Erlangen}

La distinción entre los sistemas geométricos y los sistemas métricos creó en algunas personas una interpretación errada de mi intención al separarlos. No se trataba de hacer primero una "geometría del caucho" eliminando la métrica para atender sólo a las deformaciones continuas, y buscar así los invariantes topológicos. Ciertamente no se trataba de eso. Ya había señalado Freudenthal en los años sesenta que desarmar un 
rompecabezas y volverlo a armar, por ejemplo para verificar la igualdad de área de dos triángulos o paralelogramos con la misma base y la misma altura, o para verificar el teorema de Pitágoras, era un tipo de transformación geométrica que no era topológica, pues se rompía la figura en piezas que se rearmaban en disposición diferente. Y sin embargo era una transformación que dejaba invariante el área. Pero es claro que esas transformaciones de cortar y pegar tienen detrás una métrica subyacente ("background metric" o "métrica de trasfondo") que permite la verificación de la conservación del área, así no se sepa numéricamente cuál es el área de la figura respectiva.

Hay pues que distinguir entre los aspectos métricos cualitativos y los cuantitativos. En realidad, los llamados "cualitativos" son también cuantitativos, y tal vez sea mejor distinguir más bien entre aspectos métricos cuantitativo-cualitativos o no numéricos, y aspectos métricos cuantitativos numéricos. La apreciación de que un niño es más alto que otro no necesita unidades de longitud ni números de medida, y la estimación de que una región es más grande (en área) que otra no necesita unidades de área ni números que especifiquen esas áreas.

En San Diego, California, los profesores Patrick y Alba Thompson están desarrollando investigaciones sobre el razonamiento cuantitativo de tipo cualitativo. Se está viendo muy claramente que este tipo de razonamiento es más importante que el cuantitativo numérico, y que es un prerrequisito para éste. Por ejemplo, piensen en el siguiente problema de razonamiento cuantitativo no numérico:

Carlos y David son los mejores atletas de su curso. Si apuestan una carrera en la pista atlética, llegan al tiempo. Si recorren la pista caminando rápido al estilo de la caminata olímpica, también llegan al tiempo.

Un día se les ocurre la siguiente idea: Carlos dice que él va a empezar corriendo la mitad de la distancia, y luego va a caminar la otra mitad. David dice que él va a empezar corriendo la mitad del tiempo, y luego va a caminar la otra mitad. ¿Qué cree Ud. que pasa: que también llegan al tiempo, o que Carlos gana, o que David gana? ¿Por qué piensa así?

No se trata pues de empezar con las transformaciones de tipo deformación continua sin métrica para establecer los invariantes topológicos, y luego con las proyectividades, las afinidades y las isometrías. Podemos empezar a explorar el espacio con las isometrías, y tomar sólo las afinidades que son homotecias con centro dado y factor de ampliación dado, de las que sabemos que están programadas en el cerebro de los niños. Pero de todas maneras aceptamos la métrica euclidiana de trasfondo, así no podamos asignarles todavía números específicos a las longitudes de los segmentos, a las áreas de las regiones ni a los volúmenes de los sólidos.

Es claro que hay mucho qué aprovechar del "Programa de Erlangen", pero al final de la secundaria. Explorar los cambios de escala en una sola dirección, como los mapas en relieve que exageran la altura de las montañas; o analizar las sombras de objetos que rotan y estudiar las proyectividades; o trabajar las proyecciones que llevan a la cartografía o al dibujo en perspectiva, es ciertamente muy interesante y útil para los alumnos de matemáticas, y se presta admirablemente para hacer unidades integradas de matemáticas y dibujo técnico o de matemáticas y artes plásticas. 
Pero esas posibilidades de utilizar el "Programa de Erlangen" no significan ni mucho menos que la propuesta de la geometría activa sea un intento de imponer el esquema abstracto de ese programa kleiniano a los niños de primaria y secundaria.

\section{La geometría de las transformaciones}

La importancia que se le da en la geometría activa a trasladar y rotar objetos, a reflejarlos por medio del dibujo, de los espejos y de la imaginación, es tal vez la causa de confundir la geometría activa propuesta en los nuevos programas con la llamada "geometría de las transformaciones". Ya relatamos someramente el programa de dicha geometría, propuesta por Marshall Stone y plasmada en varios textos de geometría que salieron al mercado en los Estados Unidos en la década de los setenta (por ejemplo Jacobs, [7], o Coxford y Usiskin [3]).

La base de esa propuesta de geometría de las transformaciones era la constatación de que los tres tipos de isometrías del plano son definibles en términos de uno solo: de las reflexiones. Reflexiones sobre líneas paralelas generan traslaciones, y sobre líneas que se cruzan generan rotaciones. Bastaba pues tratar las reflexiones, y sólo con ellas se podían estudiar todas las isometrías del plano.

Pero lo que era un elegante teorema matemático fue un error pedagógico, pues los niños y jóvenes entienden directamente las rotaciones y aprenden fácilmente a detectar cuáles deslizamientos son traslaciones y cuáles son combinaciones de traslaciones y rotaciones, sin necesidad de pensar en reflexiones. Estas son las más difíciles, pues no son propiamente realizables en el material concreto. Lo que algunos libros llaman "reflexiones", por ejemplo en el grupo de isometrías que dejan fijo un cuadrado, no son presentados por esos libros como reflexiones sino como rotaciones sobre ejes en el plano de la figura.

Las reflexiones no pueden hacerse con figuras hechas de material concreto: o se hacen en el cerebro, o no pueden hacerse. La ayuda de espejos, láminas semitransparentes llamadas "Mira", calcado en papel transparente o de copia, etc., pueden ayudar al cerebro a interiorizar, reversar y coordinar las reflexiones, pero no pueden suplantarlo. No me parece pues que se deba empezar por las reflexiones, ni mucho menos. $Y$ tampoco me parece que el objetivo final sea el de analizar todo el grupo de isometrías del plano. El objetivo es explorar el espacio y sus representaciones en el plano. Si para ello ayudan las rotaciones, traslaciones y reflexiones, sean bienvenidas como herramientas de trabajo. Pero no como finalidades en sí, ni como elementos de un complejo grupo de transformaciones. No faltaría más que pusiéramos como objetivo de la geometría activa el análisis del grupo de isometrías pares del plano como producto semidirecto de las traslaciones por las rotaciones.

Ni siquiera me parece que se deban definir las rotaciones o las traslaciones por medio de las reflexiones, ni por medio de nada. Me parece que se deben experimentar activamente en forma sintónica con el cuerpo, y luego en el dibujo. Ver qué tipo de movimientos conservan la dirección, cuáles la orientación en el plano o en el espacio, cuáles cambian los órdenes cíclicos de los vértices, sin definir verbalmente ninguna de estas transformaciones. En los talleres con maestros hemos comprobado la dificultad que tienen los maestros para distinguir esos aspectos activos que los niños captan inmediatamente, y la resistencia que sienten al ver que en realidad no se puede definir con palabras qué es traslación ni qué es rotación. Definirlas por medio de las reflexiones es un engaño, pues tampoco se pueden definir las reflexiones por medio de definiciones verbales. 
No estoy pues proponiendo que se trabaje la llamada "geometría de las transformaciones", sino que se trabaje la geometría por medio de aquellas transformaciones que ayuden a esa exploración activa del espacio y a desarrollar sus representaciones en la imaginación y en el plano del dibujo.

\section{Conclusión}

La necesidad de recuperar la geometría en los programas de primaria y secundaria me parece que ya va siendo aceptada por todos, inclusive por los más fieles bourbakistas de la Universidad Nacional. Esa es una de las muchas deudas que tiene el país con el Profesor Alberto Campos.

Pero no hay que negar que los argumentos de los bourbakistas contra Euclides tienen mucho fundamento. La lógica no es propiamente impecable en los Elementos. La geometría euclidiana como fuente de problemas de investigación está prácticamente agotada desde hace cien años. La falta de operatoria parece invitar a la introducción del álgebra lineal como álgebra de la geometría. Pero la conclusión no es escribir un libro de geometría sin un solo dibujo, ni reducirla al álgebra lineal, como lo hizo Dieudonné [4]. Tampoco se debe descartar el trabajo de sistematización de conceptos y de resultados que llevó a cabo tan brillantemente Euclides. Ese trabajo es un monumento de la cultura, apenas comparable con las pirámides de Egipto, las sinfonías de Beethoven o la obra de Picasso.

La propuesta de la geometría activa invita a estudiar y aprender geometría con los grandes maestros, Tales y Pitágoras, la escuela de Atenas y la de Alejandría; con los críticos de Euclides, desde Proclo y Clavius a Hilbert y Bourbaki; y con los geómetras no euclidianos, como Saccheri, Lambert, Gauss, Lobatchevsky, Bolyai, Riemann, Beltrami y Poincaré. Pero invita a estudiarla como un ejercicio activo del propio cuerpo, de la imaginación y del dibujo. Y a no dejar perder nunca el aspecto activo y dinámico para llenar el tablero de más y más figuras muertas y de símbolos estáticos. Eso no sería geometría: sería el cadáver de la geometría.

\section{Referencias}

[1] CAMPOS, Alberto. De Pitágoras a Euclides (2a. edición). Bogotá, D. E.: Universidad Nacional de Colombia-Depto. de Matemáticas y Estadística, 1984.

[2] CAMPOS, Alberto. De Euclides a Hilbert y Bourbaki. (Policopiado). Bogotá, D. E. Universidad Nacional de Colombia-Depto. de Matemáticas y Estadística, 1987.

[3] COXFORD, Arthur F., \& USISKIN, Zalman P. Geometry: A transformation approach. Palo Alto, CA, etc.: Laidlaw/Doubleday, 1975.

[4] DIEUDONNE, Jean. Algèbre linéaire et géométrie élémentaire. Paris: Hermann, 1964.

[5] HEATH, Sir Thomas L. (Ed.). The thirteen books of Euclid's Elements, 3 vols. New York: Dover, 1956.

[6] HOWSON, G., KEITEL,C., \& KILPATRICK, J. Curriculum development in mathematics. Cambridge and London: Cambridge University Press, 1981, (ver esp. pp. 102107).

[7] JACOBS, Harold R. Geometry. New York: Freeman, 1974.

[8] MINISTERIO DE EDUCACION NACIONAL. Marcos generales de los programas curriculares. Bogotá, D. E.: MEN-DGC, 1984. 
[9] MINISTERIO DE EDUCACION NACIONAL. Marco general. Matemáticas. Propuesta de programa curricular. Sexto Grado. Educación Básica Secundaria. Bogotá, D. E.: MEN-DGC, 1988.

[10] MINISTERIO DE EDUCACION NACIONAL. Marco general. Matemáticas. Propuesta de programa curricular. Séptimo Grado. Educación Básica Secundaria. Bogotá, D. E.: MEN-DGC, 1989.

[11] MINISTERIO DE EDUCACION NACIONAL. Marco general. Matemáticas. Propuesta de programa curricular. Octavo Grado. Educación Básica Secundaria. Bogotá, D. E.: MEN-DGC, 1990.

[12] PAPERT, Seymour. Mindstorms: Computers and powerful ideas. New York, NY: Basic Books, 1980. (Hay traducción castellana).

[13] RUIZ ZUÑIGA, Angel. Matemáticas y filosofla. Estudios Logicistas. San José de Costa Rica: Editorial de la Universidad de Costa Rica, 1990.

[14] VASCO, Carlos E. Sistemas geométricos. En: Un nuevo enfoque para la didáctica de las matemáticas, vol. II. Bogotá: MEN-DGC, 1988. Pp. 47-112. 